\title{
Degradability of Bosonic Gaussian channels
}

\author{
Filippo Caruso and Vittorio Giovannetti \\ NEST CNR-INFM \& Scuola Normale Superiore, Piazza dei Cavalieri 7, I-56126 Pisa, Italy
}

(Dated: September 21, 2018)

\begin{abstract}
The notion of weak-degradability of quantum channels is introduced by generalizing the degradability definition given by Devetak and Shor. Exploiting the unitary equivalence with beamsplitter/amplifier channels we then prove that a large class of one-mode Bosonic Gaussian channels are either weakly degradable or anti-degradable. In the latter case this implies that their quantum capacity $Q$ is null. In the former case instead, this allows us to establish the additivity of the coherent information for those maps which admit unitary representation with single-mode pure environment.
\end{abstract}

PACS numbers: 03.67.Hk, 42.50.Dv

\section{INTRODUCTION}

Bosonic Gaussian channels provide a realistic noise model for transmission lines which employ photons as information carriers including optical fibers, wave guides, and free-space e.m. communication. They account for all processes where the transmitted signals undergo loss, amplification, and/or squeezing transformations [1, 2]. The characterization of these transmission lines is relevant not only from a technological point of view but also from the point of view of quantum information theory where they pose some important open problems (see Ref. 3] for a review). For instance, in the context of average input photon number constraint, it is believed that the optimal (classical or quantum) communication rates [4] of such channels should be achieved by encoding messages into Gaussian input states [2, [5, 6, 7]. However, apart from the noiseless case [8], the only nontrivial map for which such conjecture has been proved is the purely lossy channel where the information carrying photons couple through beam splitters with an external vacuum state (see Ref. 9] for the classical capacity [10] case and Refs. [2, 11] for the entangled assisted capacities). Finally Bosonic Gaussian channels are generally believed [2] to provide a natural example of maps with additive properties [12] (e.g., it has been conjectured that their maximum Holevo information and minimum Rényi entropies should be additive) although only preliminary results have been obtained [9, 13, 14] so far. In this paper we discuss a property which has some relevant implications in the analysis of the quantum capacity $Q$ [15] of a large class of one-mode Bosonic Gaussian channels. Indeed we show that these maps are either weakly degradable or anti-degradable. The notions of weak-degradability and anti-degradability of a channel are introduced here as a generalization of the degradability property defined by Devetak and Shor [16]. On one hand, using an argument of Refs. [1], 17, 18], one can show that anti-degradable channels have null quantum capacity. On the other hand, it is known that channels which are degradable in the sense of Ref. [16] possess additive coherent information which allows one to express their quantum capacity, $Q$, in terms of a single-letter

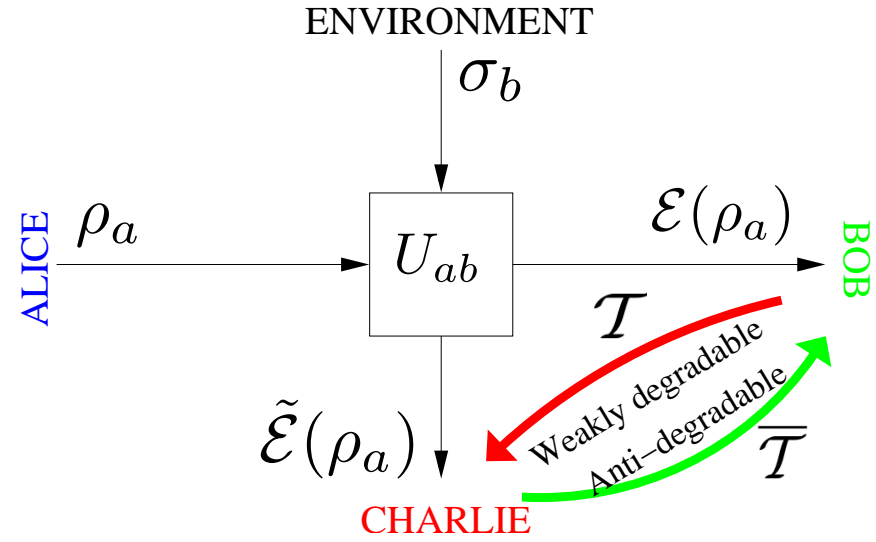

FIG. 1: Weakly degradable vs. anti-degradable channels. A channel $\mathcal{E}$ is weakly degradable if there exists a CPT map $\mathcal{T}$ which, for all input $\rho_{a}$, allows Bob to recover the environment output $\tilde{\mathcal{E}}\left[U_{a b}, \sigma_{b}\right]\left(\rho_{a}\right)$ from $\mathcal{E}\left(\rho_{a}\right)$ as in Eq. (3). A channel $\mathcal{E}$ is anti-degradable if, instead, $\mathcal{E}\left(\rho_{a}\right)$ can be recovered from $\tilde{\mathcal{E}}\left[U_{a b}, \sigma_{b}\right]\left(\rho_{a}\right)$ via a CPT transformation $\overline{\mathcal{T}}$ as in Eq. (4). Weak-degradability reduces to the degradability condition of Ref. [16] if, in Eq. (11), the environment $\sigma_{b}$ is pure.

formula. The latter is much easier to handle than its regularized version and, in some cases, allows for a complete characterization of the $Q$ (e.g., see the dephasing channel in Ref. 16] and amplitude damping channel in Ref. [17]). Weakly degradable maps do not necessarily share the above property (at present, however, we do not have a counter-example of this fact). Still, as will be clear in the following, weak-degradability provides a necessary condition for degradability and for some of the maps analyzed here the two notions coincide. In what follows, we first present the formal definitions of weak-degradability and anti-degradability (see Sec. III). Then in Sec. III we introduce one-mode Bosonic Gaussian channels and in Sec. IV we analyze their degradability properties. 


\section{WEAKLY DEGRADABLE AND ANTI-DEGRADABLE MAPS}

Consider a quantum channel described by the completely positive, trace preserving $(\mathrm{CPT})$ map $\mathcal{E}$. It can always be represented as a unitary interaction, $U_{a b}$, between the state, $\rho_{a} \in \mathcal{D}\left(\mathcal{H}_{a}\right)$, of the channel information carrier and an external environment initially prepared in a (generally mixed) state, $\sigma_{b} \in \mathcal{D}\left(\mathcal{H}_{b}\right)$, i.e

$$
\mathcal{E}\left(\rho_{a}\right)=\operatorname{Tr}_{b}\left[U_{a b}\left(\rho_{a} \otimes \sigma_{b}\right) U_{a b}^{\dagger}\right],
$$

where $\operatorname{Tr}_{b}[\ldots]$ is the partial trace over the environment and $\mathcal{D}\left(\mathcal{H}_{a, b}\right)$ are the sets of the density matrices of the system $a$ and $b$, respectively. For $\sigma_{b}$ pure Eq. (1) is equivalent to the Stinespring representation [19] of $\mathcal{E}$ and its properties are uniquely determined up to an isometry (i.e., they do not depend upon the possible choices of $U_{a b}$ and $\left.\sigma_{b}=|\psi\rangle_{b}\langle\psi|\right)$. For $\sigma_{b}$ mixed such unicity is generally lost. For this reason in the following we will write $\mathcal{E}\left[U_{a b}, \sigma_{b}\right]$ instead of $\mathcal{E}$ to make explicit which representation (11) is under consideration. We now introduce the CPT map $\tilde{\mathcal{E}}\left[U_{a b}, \sigma_{b}\right]$ which takes $\rho_{a}$ into the density matrix which describes the environment after the interaction with the carrier, i.e.,

$$
\tilde{\mathcal{E}}\left[U_{a b}, \sigma_{b}\right]\left(\rho_{a}\right) \equiv \operatorname{Tr}_{a}\left[U_{a b}\left(\rho_{a} \otimes \sigma_{b}\right) U_{a b}^{\dagger}\right],
$$

where $\operatorname{Tr}_{a}[\ldots]$ is the partial trace over the carrier (see also Fig. 11). Since we are not assuming $\sigma_{b}$ to be pure, Eq. (2) differs from the standard definition of conjugate map given in Refs. [16, 20, 21]. For this reason we name the map $\tilde{\mathcal{E}}\left[U_{a b}, \sigma_{b}\right]$ as weakly complementary or weakly conjugate of $\mathcal{E}$ with respect to the representation (11).

A channel $\mathcal{E}$ is said to be weakly degradable with respect to the representation (11) if given an unknown carrier input state $\rho_{a}$, the receiver of the messages (Bob) can reconstruct the perturbed environmental state $\tilde{\mathcal{E}}\left[U_{a b}, \sigma_{b}\right]\left(\rho_{a}\right)$ from the received density matrix $\mathcal{E}\left(\rho_{a}\right)$. This requires the existence of a third CPT transformation $\mathcal{T}: \mathcal{D}\left(\mathcal{H}_{a}\right) \rightarrow \mathcal{D}\left(\mathcal{H}_{b}\right)$ such that

$$
(\mathcal{T} \circ \mathcal{E})\left(\rho_{a}\right)=\tilde{\mathcal{E}}\left[U_{a b}, \sigma_{b}\right]\left(\rho_{a}\right),
$$

with "o" being the composition of super-operators. Analogously, generalizing [17], we say that $\mathcal{E}$ is antidegradable if, given an unknown input state $\rho_{a}$, a third party (Charlie) which is monitoring the channel environment can reconstruct Bob state $\mathcal{E}\left(\rho_{a}\right)$ from $\tilde{\mathcal{E}}\left[U_{a b}, \sigma_{b}\right]\left(\rho_{a}\right)$. As before, this requires the existence of a CPT map $\overline{\mathcal{T}}: \mathcal{D}\left(\mathcal{H}_{b}\right) \rightarrow \mathcal{D}\left(\mathcal{H}_{a}\right)$ which for all inputs gives

$$
\left(\overline{\mathcal{T}} \circ \tilde{\mathcal{E}}\left[U_{a b}, \sigma_{b}\right]\right)\left(\rho_{a}\right)=\mathcal{E}\left(\rho_{a}\right) .
$$

Finally, we say that a map $\mathcal{E}$ is weakly degradable (antidegradable) if it is weakly degradable (anti-degradable) with respect to some representation (11).

When the environmental state $\sigma_{b}$ of Eq. (11) is pure [i.e., if Eq. (1) is a Stinespring representation of $\mathcal{E}$ ] our definition of weak-degradability reduces to the degradability property introduced in Ref. [16] and implies the additivity of the coherent information of the channel. In the general case, however, weakly degradable channels need not to be degradable and Eq. (3) is only a necessary condition for degradability. Using the no-cloning theorem [22], one can prove that anti-degradable channels must have null quantum capacity $Q=0$. Indeed, assume by contradiction $Q>0$. This means that by employing sufficiently many times the map $\mathcal{E}$, Alice will be able to transfer to Bob a generic unknown state $|\psi\rangle$. However, since the channel is anti-degradable, everything Bob gets from the channel can also be reconstructed by Charlie by cascading $\tilde{\mathcal{E}}\left[U_{a b}, \sigma_{b}\right]$ with the CPT map $\overline{\mathcal{T}}$ of Eq. (4). This implies that at the end of the day both Bob and Charlie will have a copy of $|\psi\rangle$, which is impossible. Weak-degradability and anti-degradability are not mutually exclusive properties - for instance, will see that a beam splitter channel with transmissivity $1 / 2$ satisfies both Eqs. (3) and (4). Moreover, within the representation (1), the weakly complementary of $\tilde{\mathcal{E}}\left[U_{a b}, \sigma_{b}\right]$ can be identified with the original map $\mathcal{E}$ : this implies that $\mathcal{E}\left[U_{a b}, \sigma_{b}\right]$ is weakly degradable if and only if its weakly complementary $\tilde{\mathcal{E}}\left[U_{a b}, \sigma_{b}\right]$ is anti-degradable. Finally, an easy to verify but important property is the fact that maps which are unitarily equivalent to a weakly degradable channel are also weakly degradable (analogously for anti-degradability).

\section{ONE-MODE GAUSSIAN CHANNELS}

A CPT transformation $\mathcal{E}$ operating on a Bosonic mode described by the annihilation operator $a$ is said to be Gaussian if when acting on Gaussian input states $\rho_{a}$ produces output Gaussian states [1, 2]. We recall that the Gaussian states of the mode $a$ are density matrices $\rho_{a}$ whose characteristic function

$$
\chi(\mu)=\operatorname{Tr}\left[\rho_{a} \exp \left(\mu a^{\dagger}-\mu^{*} a\right)\right]
$$

is Gaussian, i.e.,

$$
\chi(\mu)=\exp \left[-\zeta \cdot \Gamma \cdot \zeta^{\dagger} / 2-\zeta_{0} \cdot \zeta^{\dagger}\right],
$$

with $\zeta=\left(\mu^{*},-\mu\right), \Gamma$ being the covariant matrix of $\rho_{a}$ and $\zeta_{0}$ being the first order momentum of the distribution 1 , [2, 3].

In the following we will focus on one-mode Gaussian channels which admit a single-mode unitary representation. They can be expressed as in Eq. (1) with $\sigma_{b}$ being a (possibly mixed) Gaussian state of a single environmental Bosonic mode described by the annihilation operator $b$. In this context $U_{a b}$ describes a linear coupling which performs the transformation [1, 2],

$$
U_{a b} \vec{v} U_{a b}^{\dagger}=A \cdot \vec{v},
$$

where $\vec{v}^{T}=\left(a, a^{\dagger}, b, b^{\dagger}\right)$ and $A$ being a $4 \times 4$ complex symplectic matrix. In particular, to preserve the commutation relation among the operators $a, a^{\dagger}, b$ and $b^{\dagger}$, 
the matrix $A$ satisfies the following constraints

$$
\sum_{j=1}^{4}(-1)^{j+1}\left|A_{i j}\right|^{2}=1
$$

for $i=1,3$ and

$$
\begin{aligned}
\sum_{j=1}^{4}(-1)^{j+1} A_{1 j} A_{3 j+(-1)^{j+1}} & =0 \\
\sum_{j=1}^{4}(-1)^{j+1} A_{1 j} A_{3 j}^{*} & =0 .
\end{aligned}
$$

Almost all the one-mode Gaussian channels can be expressed in this way. Indeed the only exception to this rule is represented by maps which are unitarily equivalent to additive-classical-Gaussian-noise channels [23, 24]. Within the single-mode unitary representation of $\mathcal{E}$, the weakly complementary map (2) of $\mathcal{E}$ is again an one-mode Gaussian channel [2, 21] which can be seen as a transformation which maps $\mathcal{D}\left(\mathcal{H}_{a}\right)$ into itself, by introducing an irrelevant isometry which exchanges $a$ and $b$ [17]. We will show that the weak-degradability of the Gaussian map $\mathcal{E}\left[U_{a b}, \sigma_{b}\right]$ with $U_{a b}$ as in Eq. (7) depends upon the real parameter,

$$
q \equiv\left|A_{11}\right|^{2}-\left|A_{12}\right|^{2}
$$

with $A_{11}$ and $A_{12}$ being elements of the matrix $A$. The quantity (10) is an invariant of the unitary representation of the map, i.e., it depends on $\mathcal{E}$ but not on the choice of $U_{a b}$ and $\sigma_{b}$. This property is discussed in details in Refs. [23, 24] (see also Ref. [14]). Here we will prove that the map $\mathcal{E}\left[U_{a b}, \sigma_{b}\right]$ is weakly degradable for $q \geqslant 1 / 2$ and anti-degradable for $q \leqslant 1 / 2$ (see Table $\llbracket$ ).

Without loss of generality, in the following we will assume $\sigma_{b}$ to have null first order momentum (it can always be compensated through a suitable unitary operator acting on the output of the channel). Another important simplification arises by considering the one-parameter family of unitaries $U_{a b}^{(k)}$ (17) associated with beam-splitter (BS) and amplifier transformations. For $k \in[0,1]$ they are characterized by the matrix

$$
A^{(k)}=\left(\begin{array}{cccc}
\sqrt{k} & 0 & -\sqrt{1-k} & 0 \\
0 & \sqrt{k} & 0 & -\sqrt{1-k} \\
\sqrt{1-k} & 0 & \sqrt{k} & 0 \\
0 & \sqrt{1-k} & 0 & \sqrt{k}
\end{array}\right),
$$

which describes superposition of the modes $a$ and $b$ at the output of a beam-splitter of transmissivity $k$. For $k \geqslant 1$ instead the $U_{a b}^{(k)}$ are characterized by the matrix

$$
A^{(k)}=\left(\begin{array}{cccc}
\sqrt{k} & 0 & 0 & -\sqrt{k-1} \\
0 & \sqrt{k} & -\sqrt{k-1} & 0 \\
0 & -\sqrt{k-1} & \sqrt{k} & 0 \\
-\sqrt{k-1} & 0 & 0 & \sqrt{k}
\end{array}\right),
$$

\begin{tabular}{|c|c|c|}
\hline \hline Value of $q$ & Equivalent map & \\
\hline \hline$q<0$ & $\tilde{\mathcal{E}}\left[1-q, \sigma_{b}^{\prime}\right]$ & $\begin{array}{c}\text { Anti-degradable } \\
(Q=0)\end{array}$ \\
& conjugate amplifier & $\begin{array}{c}\text { Anti-degradable } \\
(Q=0)\end{array}$ \\
\hline $0<q \leqslant 1 / 2$ & $\mathcal{E}\left[q, \sigma_{b}^{\prime}\right]$ & \\
& BS of transmissivity $q$ & Weakly degradable \\
$1<q$ & BS of transmissivity $q$ & $\left(q, \sigma_{b}^{\prime}\right]$ \\
amplifier & (degradable for $\sigma_{b}^{\prime}$ pure) \\
\hline
\end{tabular}

TABLE I: Weak-degradability and anti-degradability conditions for the one-mode Bosonic channel $\mathcal{E}\left[U_{a b}, \sigma_{b}\right]$. In the first column we report the value of the characteristic parameter $q$ of Eq. (10). In the second column we report the BS or amplifier map which, according to Eqs. (13) and (14), is unitarily equivalent to $\mathcal{E}$ ( $\sigma_{b}^{\prime}$ are Gaussian states obtained by properly squeezing $\sigma_{b}$ ). For $q=0$ and $q=1$ the equivalent BS or amplifier map not always exists [23], still one can show that these maps are respectively anti-degradable and weakly degradable. Channels which are anti-degradable have null quantum capacity. Those which are weakly degradable with $\sigma_{b}$ pure (i.e., degradable) have instead additive coherent information. The case $q=1 / 2$ is an example of a channel which satisfies both the weak-degradability condition (3) and the anti-degradability condition (4). This is a consequence of the symmetry of the fields emerging from the opposite output ports of a 50/50 beam-splitter.

which defines an amplification of $a$ with gain parameter $k$. Notice that in both cases Eq. (10) yields

$$
\left|A_{11}^{(k)}\right|^{2}-\left|A_{12}^{(k)}\right|^{2}=k .
$$

As discussed in Appendix $\mathrm{A}$ the corresponding maps $\mathcal{E}\left[k, \sigma_{b}\right] \equiv \mathcal{E}\left[U_{a b}^{(k)}, \sigma_{b}\right]$ and their weakly conjugate $\tilde{\mathcal{E}}\left[k, \sigma_{b}\right] \equiv \tilde{\mathcal{E}}\left[U_{a b}^{(k)}, \sigma_{b}\right]$ can be used to express a generic one-mode Gaussian channel via proper unitary transformations, with some remarkable exceptions in the case of $q=0,1$ 23]. We can, therefore, prove the weakdegradability or anti-degradability property of one-mode Gaussian maps by focusing only on the subset $\mathcal{E}\left[k, \sigma_{b}\right]$ (see Sec. IV]). Consider, in fact, a generic Gaussian map of the form $\mathcal{E}\left[U_{a b}, \sigma_{b}\right]$ with the real parameter $q$ of Eq. (10) being positive and $\neq 1$. According to Eq. (A10) of the Appendix we can write,

$$
\mathcal{E}\left[U_{a b}, \sigma_{b}\right]\left(\rho_{a}\right)=S_{a}\left(\mathcal{E}\left[k=q, \sigma_{b}^{\prime}\right]\left(\rho_{a}\right)\right) S_{a}^{\dagger},
$$

with $\sigma_{b}^{\prime} \equiv S_{b}^{\prime} \sigma_{b} S_{b}^{\prime \dagger}$ and $S_{a}, S_{b}^{\prime}$ being, respectively, unitary squeezing operators of $a$ and $b$ which depend on $A$ but not on the input state $\rho_{a}$. Since squeezed Gaussian states are Gaussian, the above expression shows that any Gaussian channel $\mathcal{E}\left[U_{a b}, \sigma_{b}\right]$ is unitarily equivalent to an amplifier channel for $q>1$ and to a BS channel for $q \in] 0,1[$. As will be shown in the next section, this implies that $\mathcal{E}\left[U_{a b}, \sigma_{b}\right]$ is anti-degradable for $\left.\left.q \in\right] 0,1 / 2\right]$ and weakly degradable for $q \geqslant 1 / 2$ and $q \neq 1$. Consider now the case of maps with $q$ of Eq. (10) being negative. Here Eq. (13) 
is replaced by

$$
\mathcal{E}\left[U_{a b}, \sigma_{b}\right]\left(\rho_{a}\right)=S_{a}\left(\tilde{\mathcal{E}}\left[1-q, \sigma_{b}^{\prime}\right]\left(\rho_{a}\right)\right) S_{a}^{\dagger},
$$

where, again, $S_{a}$ and $\sigma_{b}^{\prime}$ are, respectively, a squeezing operator and a Gaussian state [in writing Eq. (14) an isometry $a \leftrightarrow b$ is implicitly assumed]. Since $1-q>1$, Eq. (14) shows that $\mathcal{E}$ is unitarily equivalent to the weakly conjugate map of the amplifier channel $\mathcal{E}\left[1-q, \sigma_{b}^{\prime}\right]$. As discussed in the following this is equivalent to say that $\mathcal{E}\left[U_{a b}, \sigma_{b}\right]$ with negative $q$ are always anti-degradable. Finally, for $q=0$ and $q=1$ the channel is, respectively, anti-degradable and weakly degradable. The analysis of these maps is slightly more complex since it is not always possible to describe them in terms of BS/amplifier channels [23] (details are given in Ref. 24]).

\section{BS AND AMPLIFIER MAPS}

In this section we analyze the weak-degradability properties of the BS and amplifier maps.

According to the definition of the matrix $A^{(k)}$ it follows that the map $\mathcal{E}\left[k, \sigma_{b}\right]$ operates on a generic (not necessarily Gaussian) state $\rho_{a}$ by transforming its characteristic function $\chi(\mu)$ as follows:

$\chi(\mu) \rightarrow \chi^{\prime}(\mu)= \begin{cases}\chi(\sqrt{k} \mu) \xi(\sqrt{1-k} \mu) & k \in[0,1] \\ \chi(\sqrt{k} \mu) \xi\left(-\sqrt{k-1} \mu^{*}\right) & k \geqslant 1,\end{cases}$

with

$$
\xi(\mu)=\operatorname{Tr}\left[\sigma_{b} \exp \left(\mu b^{\dagger}-\mu^{*} b\right)\right],
$$

being the Gaussian characteristic function of the environment state $\sigma_{b}$ which, as previously discussed, is assumed to have null first order momentum. Analogously the weakly complementary map $\tilde{\mathcal{E}}\left[k, \sigma_{b}\right]$ produces the transformation,

$$
\chi(\mu) \rightarrow \chi^{\prime}(\mu)= \begin{cases}\chi(-\sqrt{1-k} \mu) \xi(\sqrt{k} \mu) & k \in[0,1] \\ \chi\left(-\sqrt{k-1} \mu^{*}\right) \xi(\sqrt{k} \mu) & k \geqslant 1 .\end{cases}
$$

We now show that $\mathcal{E}\left[k, \sigma_{b}\right]$ is weakly degradable for $k \geqslant$ $1 / 2$ and anti-degradable for $k \leqslant 1 / 2$.

Consider first the amplifier case where $k \geqslant 1$. To show that $\mathcal{E}\left[k, \sigma_{b}\right]$ satisfies the weak-degradability condition (3) we define the quantity $k^{\prime} \equiv(2 k-1) / k$ and notice that this is always greater than or equal to 1 . Our claim is that one can identify the map $\mathcal{T}$ of Eq. (3) with the weakly complementary map (2) of an Amplifier of gain $k^{\prime}$, i.e., $\mathcal{T}=\tilde{\mathcal{E}}\left[k^{\prime}, \sigma_{b}\right]$. This can be verified by studying how $\tilde{\mathcal{E}}\left[k^{\prime}, \sigma_{b}\right] \circ \mathcal{E}\left[k, \sigma_{b}\right]$ acts on a generic state $\rho_{a}$. Combining Eqs. (15), (17) it follows that the characteristic function $\chi(\mu)$ of $\rho_{a}$ is transformed into

$$
\begin{aligned}
\chi(- & \left.\sqrt{k\left(k^{\prime}-1\right)} \mu^{*}\right) \xi\left(\sqrt{\left(k^{\prime}-1\right)(k-1)} \mu\right) \xi\left(\sqrt{k^{\prime}} \mu\right) \\
& =\chi\left(-\sqrt{k\left(k^{\prime}-1\right)} \mu^{*}\right) \xi\left(\sqrt{\left(k^{\prime}-1\right)(k-1)+k^{\prime}} \mu\right) \\
& =\chi\left(-\sqrt{k-1} \mu^{*}\right) \xi(\sqrt{k} \mu),
\end{aligned}
$$

where we used the properties of the Gaussian function $\xi$ and the identity $k\left(k^{\prime}-1\right)=k-1$. By comparison with Eq. (17), we notice that $\tilde{\mathcal{E}}\left[k^{\prime}, \sigma_{b}\right] \circ \mathcal{E}\left[k, \sigma_{b}\right]$ operates on $\rho_{a}$ as $\tilde{\mathcal{E}}\left[k, \sigma_{b}\right]$. Since this is true for all $\rho_{a}$ we get

$$
\tilde{\mathcal{E}}\left[k, \sigma_{b}\right]=\tilde{\mathcal{E}}\left[k^{\prime}, \sigma_{b}\right] \circ \mathcal{E}\left[k, \sigma_{b}\right]
$$

proving the thesis.

Consider now the BS case where $k \in[0,1]$. Here we distinguish two different regimes. For $k \in[1 / 2,1]$ the channel $\mathcal{E}\left[k, \sigma_{b}\right]$ is still weakly degradable and satisfies Eq. (19), the only difference being that now $\tilde{\mathcal{E}}\left[k^{\prime}, \sigma_{b}\right]$ represents the weakly complementary of a beam-splitter map of transmissivity $k^{\prime}=(2 k-1) / k \in[0,1]$. The formal proof goes as in Eq. (18), which now becomes

$$
\begin{aligned}
\chi( & \left.-\sqrt{k\left(1-k^{\prime}\right)} \mu\right) \xi\left(-\sqrt{\left(1-k^{\prime}\right)(1-k)} \mu\right) \xi\left(\sqrt{k^{\prime}} \mu\right) \\
& =\chi\left(-\sqrt{k\left(1-k^{\prime}\right)} \mu\right) \xi\left(\sqrt{\left(1-k^{\prime}\right)(1-k)+k^{\prime}} \mu\right) \\
& =\chi(-\sqrt{1-k} \mu) \xi(\sqrt{k} \mu) .
\end{aligned}
$$

For $k \in[0,1 / 2]$ instead we can show that $\mathcal{E}\left[k, \sigma_{b}\right]$ is anti-degradable by observing that it satisfies the condition (4) with $\overline{\mathcal{T}}$ being the weakly complementary $\tilde{\mathcal{E}}\left[k^{\prime \prime}, \sigma_{b}\right]$ of a BS channel of transmissivity $k^{\prime \prime}=(1-2 k) /(1-k) \in$ $[0,1]$, i.e.,

$$
\mathcal{E}\left[k, \sigma_{b}\right]=\tilde{\mathcal{E}}\left[k^{\prime \prime}, \sigma_{b}\right] \circ \tilde{\mathcal{E}}\left[k, \sigma_{b}\right]
$$

The proof is again obtained through Eqs. (15) and (17) by showing that the transformations on a generic $\chi(\mu)$ induced by $\tilde{\mathcal{E}}\left[k^{\prime \prime}, \sigma_{b}\right] \circ \tilde{\mathcal{E}}\left[k, \sigma_{b}\right]$ and by $\mathcal{E}\left[k, \sigma_{b}\right]$ coincide.

Indeed, one has

$$
\begin{aligned}
\chi & \left(\sqrt{(1-k)\left(1-k^{\prime \prime}\right)} \mu\right) \xi\left(\sqrt{\left(1-k^{\prime \prime}\right) k} \mu\right) \xi\left(\sqrt{k^{\prime \prime}} \mu\right) \\
& =\chi\left(\sqrt{(1-k)\left(1-k^{\prime \prime}\right)} \mu\right) \xi\left(\sqrt{\left(1-k^{\prime \prime}\right) k+k^{\prime \prime}} \mu\right) \\
& =\chi(\sqrt{k} \mu) \xi(\sqrt{1-k} \mu) .
\end{aligned}
$$

\section{CONCLUSIONS}

A new property of quantum channels (i.e., weakdegradability) is introduced by exploiting a more "physical" picture of the noise evolution (i.e., interaction with a thermal-like environment). We prove that with the exception of the additive-classical-Gaussian-noise channels [23], all one-mode Gaussian maps are weakly degradable or anti-degradable. In the latter case this implies that their quantum capacity $Q$ must be null. In the former case instead this yields the additivity of their coherent information under the condition that the representation (1) upon which weak-degradability was derived, possesses single-mode pure environmental state. For the sake of completeness we mention that after the submission of our manuscript, Wolf et al. posted a couple of interesting papers [26] where, adopting an approach similar to ours, the quantum capacities of several quantum 
channels were explicitly solved (including the one-mode Gaussian channels which here we show to have additive coherent information).

\section{Acknowledgments}

We thank Professor A. S. Holevo for pointing out a flaw in our original argument. This led us to the definition of weak-degradability and helped us in clarifying the possibility of describing almost all one-mode Gaussian maps in terms of single-mode environment representations. We also thank Professor R. Fazio for comments. This work was supported by the Quantum Information research program of Centro di Ricerca Matematica Ennio De Giorgi of the Scuola Normale Superiore.

\section{APPENDIX A: DECOMPOSITION RULES}

Here we give an explicit derivation of the decomposition rules (13) and (14) which allow us to express any generic one-mode Gaussian map with $q \neq 0,1$ in terms of BS or amplifier channels. For the sake of clarity we will analyze separately the cases $q \in] 0,1[, q>1$ and $q<0$.

\section{Maps with $q \in] 0,1[$}

Consider first the case of one-mode Gaussian channel of the form $\mathcal{E}\left[U_{a b}, \sigma_{b}\right]$ with the real parameter $q$ of Eq. (10) being positive and smaller than 1 . Under this condition, apart from redefining the phasis of $a$ and $b$, the elements $A_{1 j}$ of the matrix (7) can be parameterized as follows

$$
\begin{aligned}
& A_{11}=\sqrt{q} \cosh r, \\
& A_{12}=\sqrt{q} e^{i \varphi} \sinh r, \\
& A_{13}=-\sqrt{1-q} \cosh s, \\
& A_{14}=-\sqrt{1-q} e^{i \psi} \sinh s,
\end{aligned}
$$

where $r, s, \varphi$, and $\psi$ are real quantities and where the last two expressions come from the constraint (8). Let us then introduce the (unitary) squeezing transformations [25]:

$$
\begin{aligned}
S_{a}(r ; \varphi) a S_{a}^{\dagger}(r ; \varphi) & =a \cosh r+e^{i \varphi} a^{\dagger} \sinh r \\
S_{b}(s ; \psi) b S_{b}^{\dagger}(s ; \psi) & =b \cosh s+e^{i \psi} b^{\dagger} \sinh s .
\end{aligned}
$$

On one hand, they allow us to write

$$
\begin{aligned}
\left(S_{a}^{\dagger} \otimes S_{b}^{\dagger}\right) a^{\prime}\left(S_{a} \otimes S_{b}\right) & =\sqrt{q} a-\sqrt{1-q} b \\
& =U_{a b}^{(q)} a\left[U_{a b}^{(q)}\right]^{\dagger}
\end{aligned}
$$

where $U_{a b}^{(q)}$ is the BS transformation defined as in Eq. (11) while $a^{\prime}=U_{a b} a U_{a b}^{\dagger}$ represents the evolution of $a$ under the unitary $U_{a b}$ of $\mathcal{E}\left[U_{a b}, \sigma_{b}\right]$. On the other hand, we get

$\left(S_{a}^{\dagger} \otimes S_{b}^{\dagger}\right) b^{\prime}\left(S_{a} \otimes S_{b}\right)=\bar{A}_{21} a+\bar{A}_{22} a^{\dagger}+\bar{A}_{23} b+\bar{A}_{24} b^{\dagger} 1$, with $b^{\prime}=U_{a b} b U_{a b}^{\dagger}$ and with $\bar{A}_{2 j}$ being complex parameters which satisfies the symplectic conditions analogous to those of Eqs. (8) and (9), i.e.,

$$
\begin{aligned}
\left|\bar{A}_{21}\right|^{2}-\left|\bar{A}_{22}\right|^{2}+\left|\bar{A}_{23}\right|^{2}-\left|\bar{A}_{24}\right|^{2} & =1 \\
\sqrt{q} \bar{A}_{21}-\sqrt{1-q} \bar{A}_{23} & =0 \\
\sqrt{q} \bar{A}_{22}-\sqrt{1-q} \bar{A}_{24} & =0
\end{aligned}
$$

Equation (A4) can be cast in a more compact form by properly parameterizing the $\bar{A}_{2 j}$;

$$
\begin{aligned}
& \bar{A}_{21}=\sqrt{1-q} \cosh (t) e^{i \phi}, \\
& \bar{A}_{22}=\sqrt{1-q} \sinh (t) e^{i \phi^{\prime}}, \\
& \bar{A}_{23}=\sqrt{q} \cosh (t) e^{i \phi}, \\
& \bar{A}_{24}=\sqrt{q} \sinh (t) e^{i \phi^{\prime}},
\end{aligned}
$$

with $t, \phi$, and $\phi^{\prime}$ real. This yields

$$
\left(S_{a}^{\dagger} \otimes S_{b}^{\dagger}\right) b^{\prime}\left(S_{a} \otimes S_{b}\right)=e^{i \phi} U_{a b}^{(q)}\left(S_{b}^{\prime} b S_{b}^{\dagger \dagger}\right)\left[U_{a b}^{(q)}\right]^{\dagger}
$$

where $S_{b}^{\prime} \equiv S_{b}\left(t, \phi^{\prime}-\phi\right)$ is a squeezing operator (A4) acting on $b$ and where $U_{a b}^{(q)}$ is the BS unitary coupling of Eq. (A3). By absorbing the phase $\phi$ into the definition of $b^{\prime}$ and by noticing that $S_{b}^{\prime}$ does not affect $a$, Eqs. (A3), (A7), and (7) give

$$
U_{a b} \vec{v} U_{a b}^{\dagger}=\left(S_{a} \otimes S_{b}\right) U_{a b}^{(q)} S_{b}^{\prime} \vec{v} S_{b}^{\dagger}\left[U_{a b}^{(q)}\right]^{\dagger}\left(S_{a} \otimes S_{b}\right)^{\dagger}
$$

which allows us to decompose $U_{a b}$ as the following product:

$$
U_{a b}=\left(S_{a} \otimes S_{b}\right) U_{a b}^{(q)} S_{b}^{\prime}
$$

Replacing this into Eq. (1) we finally get

$$
\begin{aligned}
\mathcal{E}\left[U_{a b}, \sigma_{b}\right]\left(\rho_{a}\right) & =S_{a} \operatorname{Tr}_{b}\left[S_{b} U_{a b}^{(q)}\left(\rho_{a} \otimes \sigma_{b}^{\prime}\right)\left[U_{a b}^{(q)}\right]^{\dagger} S_{b}^{\dagger}\right] S_{a}^{\dagger} \\
& =S_{a} \operatorname{Tr}_{b}\left[U_{a b}^{(q)}\left(\rho_{a} \otimes \sigma_{b}^{\prime}\right)\left[U_{a b}^{(q)}\right]^{\dagger}\right] S_{a}^{\dagger} \\
& =S_{a}\left(\mathcal{E}\left[k=q, \sigma_{b}^{\prime}\right]\left(\rho_{a}\right)\right) S_{a}^{\dagger} .
\end{aligned}
$$

In this expression the $S_{a}$ was brought out of the trace since it is acting on $a$. Vice versa, $S_{b}$ has been simplified by exploiting the invariance of the trace under unitary transformation. Finally, the Gaussian state $\sigma_{b}^{\prime}$ is the squeezed version under $S_{b}^{\prime}$ of the environmental state $\sigma_{b}$, i.e.,

$$
\sigma_{b}^{\prime} \equiv S_{b}^{\prime} \sigma_{b} S_{b}^{\dagger}
$$

Equation (A10) shows that, for $q \in] 0,1[$ the map $\mathcal{E}\left[U_{a b}, \sigma_{b}\right]$ is unitary equivalent to the BS channel $\mathcal{E}[k=$ $\left.q, \sigma_{b}^{\prime}\right]$ 


\section{Maps with $q>1$}

For $q$ greater than one Eqs. (A9) and (A10) still hold: the only difference being that now $U_{a b}^{(q)}$ represents an amplifier map defined by the matrix of Eq. (12). This can be shown following the same derivation of the case $q \in$ ] $0,1[$ by replacing the parameterizations (A1) and (A6) with

$$
\begin{aligned}
& A_{11}=\sqrt{q} \cosh r \\
& A_{12}=\sqrt{q} e^{i \varphi} \sinh r \\
& A_{13}=-\sqrt{q-1} e^{-i \psi} \sinh s \\
& A_{14}=-\sqrt{q-1} \cosh s
\end{aligned}
$$

and

$$
\begin{aligned}
& \bar{A}_{21}=-\sqrt{q-1} \sinh (t) e^{i \phi^{\prime}}, \\
& \bar{A}_{22}=-\sqrt{q-1} \cosh (t) e^{i \phi}, \\
& \bar{A}_{23}=\sqrt{q} \cosh (t) e^{i \phi}, \\
& \bar{A}_{24}=\sqrt{q} \sinh (t) e^{i \phi^{\prime}} .
\end{aligned}
$$

\section{Maps with $q<0$}

To analyze the channels $\mathcal{E}\left[U_{a b}, \sigma_{b}\right]$ with $q$ negative it is useful to introduce a isometry $\Xi_{a b}=\Xi_{a b}^{\dagger}$ which transforms $a$ in $b$ and vice versa while leaving the vacuum state invariant, i.e., $\Xi_{a b} a \Xi_{a b}=b, \Xi_{a b} b \Xi_{a b}=a$, and $\Xi_{a b}|\varnothing\rangle=|\varnothing\rangle$. This is a unitary transformation which for any bounded operator $\Theta_{a b}$ on $\mathcal{H}_{a} \otimes \mathcal{H}_{b}$ satisfies the identity

$$
\operatorname{Tr}_{b}\left[\Xi_{a b} \Theta_{a b} \Xi_{a b}\right] \otimes \mathbb{1}_{b}=\Xi_{a b}\left(\mathbb{1}_{a} \otimes \operatorname{Tr}_{a}\left[\Theta_{a b}\right]\right) \Xi_{a b} .
$$

Consider, then, the unitary transformation $\Xi_{a b} U_{a b}$ with $U_{a b}$ being the unitary coupling associated with $\mathcal{E}\left[U_{a b}, \sigma_{b}\right]$. From Eq. (7) it follows

$$
\left(\Xi_{a b} U_{a b}\right) \vec{v}\left(U_{a b}^{\dagger} \Xi_{a b}\right)=\tilde{A} \cdot \vec{v}
$$

where $\tilde{A}$ is a $4 \times 4$ matrix which is obtained by shifting by 2 the columns of the matrix $A$ which describes the unitary $U_{a b}$, i.e., $\tilde{A}_{i j}=A_{i, j \oplus 2}$ where $\oplus$ represents the sum modulus 4 . From the constraint (8) it then follows that the coefficient (10) of $\tilde{A}$ is greater than 1, i.e.,

$$
\begin{aligned}
\tilde{q} & =\left|\tilde{A}_{11}\right|^{2}-\left|\tilde{A}_{12}\right|^{2}=\left|A_{13}\right|^{2}-\left|A_{14}\right|^{2} \\
& =1-\left(\left|A_{11}\right|^{2}-\left|A_{12}\right|^{2}\right)=1-q>1 .
\end{aligned}
$$

We can then use the previous section to show that there exist squeezing transformations $S_{a}, S_{b}$, and $S_{b}^{\prime}$ which allows us to write $\Xi_{a b} U_{a b}=\left(S_{a} \otimes S_{b}\right) U_{a b}^{(\tilde{q})} S_{b}^{\prime}$ with $U_{a b}^{(\tilde{q})}$ being an Amplifier coupling (12). Therefore, we get

$$
U_{a b}=\Xi_{a b}\left(S_{a} \otimes S_{b}\right) U_{a b}^{(\tilde{q})} S_{b}^{\prime} .
$$

Exploiting the identity (A14) this yields

$$
\begin{aligned}
\mathcal{E}\left[U_{a b}, \sigma_{b}\right]\left(\rho_{a}\right) \otimes \mathbb{1}_{b} & =\operatorname{Tr}_{b}\left[\Xi_{a b}\left(S_{a} \otimes S_{b}\right) U_{a b}^{(\tilde{q})} S_{b}^{\prime}\left(\rho_{a} \otimes \sigma_{b}\right) S_{b}^{\prime \dagger}\left[U_{a b}^{(\tilde{q})}\right]^{\dagger}\left(S_{a} \otimes S_{b}\right)^{\dagger} \Xi_{a b}\right] \otimes \mathbb{1}_{b} \\
& =\Xi_{a b}\left(\mathbb{1}_{a} \otimes \operatorname{Tr}_{a}\left[\left(S_{a} \otimes S_{b}\right) U_{a b}^{(\tilde{q})} \times\left(\rho_{a} \otimes \sigma_{b}^{\prime}\right)\left[U_{a b}^{(\tilde{q})}\right]^{\dagger}\left(S_{a} \otimes S_{b}\right)^{\dagger}\right]\right) \Xi_{a b} \\
& =\Xi_{a b}\left(\mathbb{1}_{a} \otimes S_{b} \operatorname{Tr}_{a}\left[U_{a b}^{(\tilde{q})}\left(\rho_{a} \otimes \sigma_{b}^{\prime}\right)\left[U_{a b}^{(\tilde{q})}\right]^{\dagger}\right] S_{b}^{\dagger}\right) \Xi_{a b}=\Xi_{a b}\left(\mathbb{1}_{a} \otimes S_{b} \tilde{\mathcal{E}}\left[1-q, \sigma_{b}^{\prime}\right]\left(\rho_{a}\right) S_{b}^{\dagger}\right) \Xi_{a b},
\end{aligned}
$$

where we used the fact that $\operatorname{Tr}_{a}\left[U_{a b}^{(\tilde{q})}\left(\rho_{a} \otimes \sigma_{b}^{\prime}\right)\left[U_{a b}^{(\tilde{q})}\right]^{\dagger}\right]$ is the weakly complementary channel $\tilde{\mathcal{E}}\left[\tilde{q}, \sigma_{b}^{\prime}\right]$ of an amplifier with coupling $U_{a b}^{(\tilde{q})}$ and the identity $\tilde{q}=1-q$.
Finally, the above expression can be cast in the less formal but certainly simpler form (14) where the isometry $\Xi_{a b}$ is implicitly assumed.
[1] A. S. Holevo, Probabilistic Aspects of Quantum Theory (North-Holland, Amsterdam, 1982).

[2] A. S. Holevo and R. F. Werner, Phys. Rev. A 63032312 (2001).

[3] J. Eisert and M. M. Wolf, e-print quant-ph/0505151 A. Ferraro, S. Olivares, and M. G. A. Paris, Gaussian states in quantum information (Bibliopolis, Napoli, 2005).

[4] C. H. Bennett and P. W. Shor, IEEE Trans. Inf. Theory 44, 2724 (1998).

[5] M. J. W. Hall and M. J. O'Rourke, Quantum Opt. 5 161, (1993); M. J. W. Hall, Phys. Rev. A 50, 3295 (1994).

[6] J. H. Shapiro, V. Giovannetti, S. Guha, S. Lloyd, L. Mac- 
cone, and B. J. Yen AIP Conf. Proc. 734, 15 (2004).

[7] V. Giovannetti, S. Guha, S. Lloyd, L. Maccone, and J. H. Shapiro, Phys. Rev. A 70, 032315 (2004); V. Giovannetti, S. Lloyd, L. Maccone, J. H. Shapiro, and B. J. Yen, ibid. 70, 022328 (2004).

[8] C. M. Caves and P. D. Drummond, Rev. Mod. Phys. 66, 481 (1994); H. P. Yuen and M. Ozawa, Phys. Rev. Lett. 70363 (1993).

[9] V. Giovannetti, S. Guha, S. Lloyd, L. Maccone, J. H. Shapiro, and H. P. Yuen, Phys. Rev. Lett. 92, 027902 (2004).

[10] A. S. Holevo, IEEE Trans. Inf. Theory 44, 269 (1998); P. Hausladen, R. Jozsa, B. Schumacher, M. Westmoreland and W. K. Wootters, Phys. Rev. A 54, 1869 (1996); B. Schumacher and M. D. Westmoreland, ibid. 56, 131 (1997).

[11] V. Giovannetti, S. Lloyd, L. Maccone and P. W. Shor, Phys. Rev. Lett. 91, 047901 (2003); Phys. Rev. A 68, 062323 (2003).

[12] P. W. Shor, Commun. Math. Phys. 246, 453 (2004).

[13] V. Giovannetti and S. Lloyd, Phys. Rev. A 69, 062307 (2004).

[14] A. Serafini, J. Eisert, and M. M. Wolf, Phys. Rev. A 71 012320 (2005).
[15] S. Lloyd, Phys. Rev. A 55, 1613 (1997); H. Barnum, M. A. Nielsen, and B. Schumacher, ibid. 57, 4153 (1998); I. Devetak, IEEE Trans. Inform. Theory 51, 44 (2005).

[16] I. Devetak and P. W. Shor, Commun. Math. Phys. 256, 287 (2005).

[17] V. Giovannetti and R. Fazio, Phys. Rev. A 71, 032314 (2005).

[18] C. H. Bennett, D. P. DiVincenzo, J.A. Smolin, Phys. Rev. Lett. 783217 (1997).

[19] W. F. Stinespring, Proc. Am. Math. Soc. 6, 211 (1955).

[20] C. King, K. Matsumoto, M. Nathanson, and M. B. Ruskai, e-print quant-ph/0509126.

[21] A. S. Holevo, e-print quant-ph/0509101

[22] W. K. Wootters and W. H. Zurek, Nature 299, 802 (1982).

[23] A. S. Holevo, e-print quant-ph/0607051

[24] F. Caruso, V. Giovannetti, and A. S. Holevo, New J. Phys. 8, 310 (2006).

[25] D. F. Walls and G. J. Milburn, Quantum optics (Springer Verlag, Berlin 1994).

[26] M. M. Wolf et al., e-prints quant-ph/0606132 and quant-ph/0607070. 